\title{
Range-Error Compensation for a Troposphere With Exponentially Varying Refractivity ${ }^{1}$
}

\author{
J. J. Freeman \\ Contribution From J. J. Freeman Associates, Inc., 8416 Georgia Avenue, Silver Spring, Md.
}

(Received June 12, 1962)

An explicit formula for tropospheric range error is derived for a troposphere with a spherically symmetric refractivity which varies exponentially with height. The correction is given as function of surface refractivity and ray elevation angle, and its accuracy and limitations are discussed.

In the long-range tracking of an object in space by an observer on the earth, systematic errors are introduced by the earth's atmosphere, most of the contribution arising from the troposphere at microwave frequencies. This paper derives an explicit analytic expression for the tropospheric range error for a standard atmosphere whose refractivity is exponentially distributed with height.

If one measures the delay

$$
\tau=1 / c \int_{0}^{1} n d s
$$

incurred by a wave between point (0), on the earth's surface, and point (1), above the troposphere, then the measured range, $R_{e}$, between (0) and (1) is defined by

$$
R_{e}=c \tau=\int_{0}^{1} n d s
$$

the integral extending over the curved ray path. Figure 1 illustrates the geometry involved. Here $c$ is the velocity of light in vacuo, and $n$ is the index of refraction. The true or slant range between $(0)$ and (1) is

$$
R_{0}=\int_{0}^{1} d s
$$

the integral being taken along the geometric line joining (0) and (1). The error arising from the refractivity is

$$
\Delta R=\int_{0}^{1} n d s-R_{0}
$$

The range error, $\Delta R$, arises from (a) the increase in path length due to the curvature of the ray path, and (b) the decreased propagation velocity.

It can be shown that the error arising from the increase in path length is of the second order in

1 This work was supported by the Goddard Space Center, NASA, contract NAS $5-1404$. $(n-1)$, whereas the error arising from the decrease in wave velocity is of the first order in $(n-1)$, so that as a first approximation,

$$
\Delta_{1} R=\int_{0}^{1}(n-1) d s,
$$

where the integral is now taken along the slant range path. This mathematical stratagem permits one to integrate the value of $\Delta_{1} R$ directly when $n$ has a simple functional dependence on height. The validity of this approximation is evident from table 1 which enumerates the range error, $\Delta R$ (column 2), the excess of ray path over geometric path, $\Delta L$ (column 3) and percent range error due to the excess of path length, $100 \Delta L / \Delta R$ (column 4), as a function

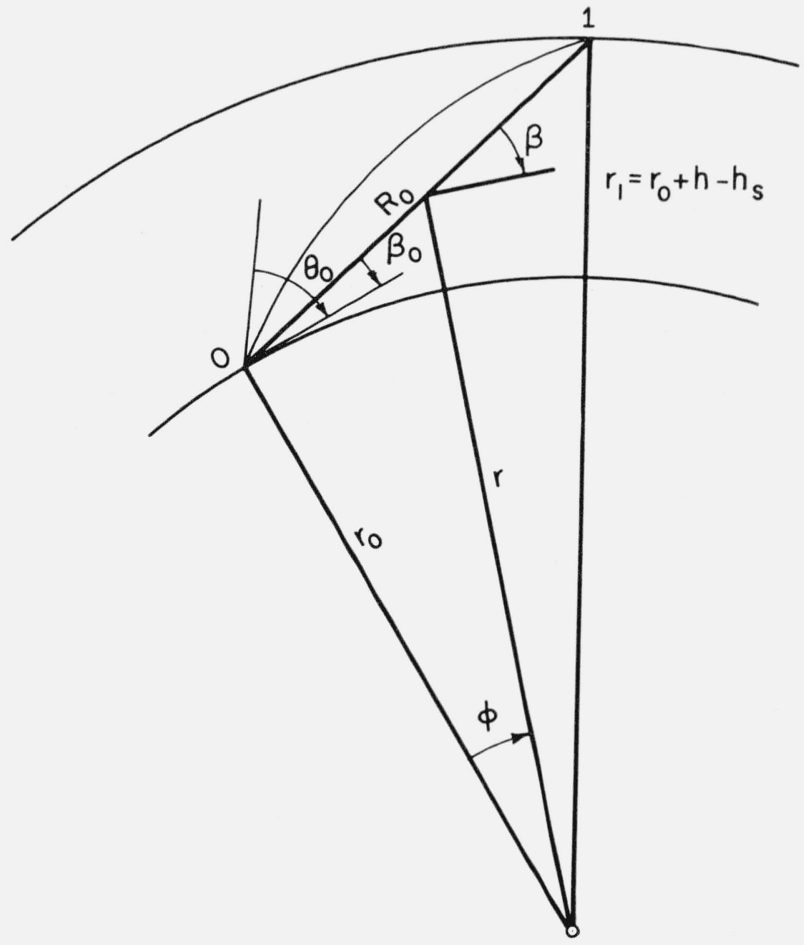

Figure 1. Geometry of ray path. 
of ray initial elevation angle, $\theta_{0}$. These values were computed from ray tracings for a typical surface refractivity $N_{s}=\left(n_{s}-1\right) \times 10^{6}=313$ for an exponential reference atmosphere and are taken from an article [1959a] by Bean and Thayer.

Table 1 shows that even for the worst possible case, only 8 percent of the total range error arises from the excess of the ray over the geometric path length, and that this percentage decreases rapidly as the initial ray elevation angle increases, becoming negligible for initial ray elevations of a few degrees.

TABLE 1.

\begin{tabular}{|c|c|c|c|}
\hline $\begin{array}{l}\theta_{0} \text {, Initial } \\
\text { ray } \\
\text { elevation }\end{array}$ & $\begin{array}{l}\Delta R=\text { range } \\
\quad \text { error }\end{array}$ & $\begin{array}{l}\text { Excess of ray } \\
\text { path over } \\
\text { geometric path }\end{array}$ & $100 \Delta L / \Delta R$ \\
\hline $\begin{array}{r}m r \\
0 \\
8 \\
15 \\
30 \\
65 \\
100 \\
200 \\
400\end{array}$ & $\begin{array}{c}m \\
104 \\
81.4 \\
68.1 \\
49.7 \\
29.5 \\
20.7 \\
10.9 \\
5.6\end{array}$ & $\begin{array}{l}m \\
9 \\
4.8 \\
3.2 \\
1.3 \\
0.3 \\
0.1\end{array}$ & $\begin{array}{l}8.2 \\
5.8 \\
4.4 \\
2.6 \\
1.0 \\
0.5\end{array}$ \\
\hline
\end{tabular}

$\Delta R$ is not very sensitive to the distribution of $n-1=N \times 10^{-6}$ with height, since only the integrated effect or the first moment of $N$ comes into consideration. Also, it has been found [Bean and Thayer, 1959b; Hoehndorf, 1961] that the average decay of $N$ with height is nearly exponential, so that the distribution of $N$ may be approximately described by

$$
N=N_{s} \exp \left\{-c\left(h-h_{s}\right)\right\},
$$

where $N_{s}$ is the surface refractivity at a locality which is height $h_{s}$ above sea level. Further extensive analyses [Bean and Thayer, $1951 \mathrm{a}$ and b] have shown that there is a very strong correlation between $N_{s}, \Delta N$, and $h_{s}$ of (3), where $\Delta N$ is the decrease from surface refractivity observed $1 \mathrm{~km}$ above the surface. Utilizing this mean relationship between $\Delta N$ and $N_{s}$, Bean and Thayer have published tables giving $c$ as a function of $N_{s}$. Other methods of determining the value of the parameter $c$ which will best enable one to predict the tropospheric range error have been explored. However, if one assumes that the distribution of $N$ is exponential with height, and if one utilizes the approximation given in (2), then (2) may be integrated explicitly as is shown in the appendix, giving

$$
\begin{gathered}
\Delta_{1} R=10^{-6} \int_{0}^{1} N d s \\
=\frac{10^{-6} N_{s} \sqrt{\pi} g e^{g^{2}}}{c \sin \beta_{0}}\left[\operatorname{erf}\left(R_{0} \cos \beta_{0} \sqrt{\frac{c}{2 r_{0}}}+g\right)-\operatorname{erf}(g)\right] \\
\operatorname{erf} g=\frac{2}{\sqrt{\pi}} \int_{0}^{g} e^{-x^{2}} d x
\end{gathered}
$$

\begin{tabular}{|c|c|c|c|c|}
\hline $\begin{array}{l}\text { Ray ele- } \\
\text { vation, } \theta_{0}\end{array}$ & $\begin{array}{c}\text { Slant } \\
\text { elevation, } \\
\boldsymbol{\beta}_{0}\end{array}$ & $\Delta_{1} R$ & $\Delta R$ & $10^{-6} N_{s} / c \sin \beta_{0}$ \\
\hline $\begin{array}{r}m r \\
0 \\
8 \\
15 \\
30 \\
65 \\
100 \\
200 \\
400\end{array}$ & $\begin{array}{c}m r \\
\\
6.324 \\
23.51 \\
61.04 \\
97.21 \\
198.5 \\
399.3\end{array}$ & $\begin{array}{r}m \\
\\
71.3 \\
50.7 \\
29.5 \\
20.4 \\
10.9 \\
5.6\end{array}$ & $\begin{array}{c}m \\
104 \\
81.4 \\
68.1 \\
49.7 \\
29.5 \\
20.7 \\
10.9 \\
5.6\end{array}$ & $\begin{array}{c}m \\
\\
272 \\
145 \\
72.4 \\
33.4 \\
21.8 \\
10.9 \\
5.6\end{array}$ \\
\hline
\end{tabular}

TABLE 2. Comparison of $\Delta_{1} \mathrm{R}$ and $\Delta \mathrm{R}$ for $\mathrm{N}_{s}=313$ for target above troposphere

and

$$
g=\tan \beta_{0} \sqrt{c r_{0} / 2} .
$$

Here $r_{0}$ is the distance from earth's center to ground, that is, the earth's radius plus height above sea level, $\beta_{0}$ is the slant range elevation angle at ground.

For the point (1) situated above the troposphere the range of integration of (4) may be extended to infinity with negligible error, yielding

$$
\Delta_{1} R=10^{-6} \int_{0}^{1} N d s=\frac{10^{-6} N_{s} \sqrt{\pi} g e^{g 2}}{c \sin \beta_{0}}[1-\operatorname{erf}(g)] .
$$

For $g$ large, (4) approaches the plane earth approximation,

$$
\Delta_{2} R=\frac{10^{-6} N_{0}}{c \sin \beta_{0}}
$$

which, of course, does not converge at $\beta_{0}=0$, whereas (6) does.

Table 2 enumerates the different computed range corrections, where $\Delta_{1} R$ is the approximate correction (6), $\Delta R$ the true correction, and the last column is the plane earth correction.

Comparing $\Delta_{1} R$ and $\Delta R$, it is apparent that (6) is a good approximation to the tropospheric error one would compute for an exponential atmosphere from a ray tracing. Its simple form suggests that it will be valuable for predicting tropospheric range error from a knowledge of only the surface conditions. The question of how to determine the decay constant, $c$, to give accurate predictions and the probable error of such predictions will be reported in a future paper.

It is worth remarking that for

$$
N=N_{s} \exp \left\{-c\left(h-h_{s}\right)-d\left(h-h_{s}\right)^{2}\right\}
$$

the expression for range error, $\Delta_{1} R$, turns out to be precisely (4) and (6), except that now one has

$$
g=\sqrt{\frac{2 \sin \beta_{0}}{\frac{2 c \cos ^{2} \beta_{0}}{r_{0}}+4 d \sin ^{2} \beta_{0}}} .
$$


The author thanks B. R. Bean and G. D. Thayer for making a number of extremely helpful suggestions. He also thanks Mrs. Charlotte McFaul for her computational assistance.

\section{Appendix}

Consider $\int_{0}^{1} n d s$. Since the difference between the distance along the ray path and that along the slant line path is negligible (see fig. 1),

$\int_{0}^{1} n d s=\int_{0}^{1} d s+10^{-6} \int_{0}^{1} N d s=R_{0}+10^{-6} \int_{0}^{1} N d s$,

where $n=1+N \times 10^{-6}, R_{0}$ is the slant range, and the integrals on the right hand side are taken along the slant range path. Along this geometric line,

$$
\frac{d \varphi}{d r}=\frac{r_{0} \cos \beta_{0}}{r \sqrt{r^{2}-r_{0}^{2} \cos ^{2} \beta}}
$$

where $r, \varphi$ are polar coordinates with earth's center as origin, and $\beta$ designates the angle between slant path direction and the tangent to the radial, that is, the elevation angle. $r_{0}, \beta_{0}$ pertain to the initial path point. Then, letting $K=r_{0} \cos \beta_{0}$,

$$
s(r)=\sqrt{r^{2}-K^{2}}-\sqrt{r_{0}^{2}-K^{2}}
$$

is the distance along the geometric path. Expressing $r$ in terms of $s$, and letting $h^{\prime}=h-h_{s}$ designate height above the earth's surface,

$$
r=r_{0}+h^{\prime}=r_{0}\left[1+\frac{s^{2}}{r_{0}^{2}}+\frac{2 s \sin \beta_{0}}{r_{0}}\right]^{\frac{1}{2}} .
$$

Expanding in terms of $s / r_{0}$ to terms of the second order,

$$
h^{\prime}=s \sin \beta_{0}+\frac{s^{2}}{2 r_{0}} \cos ^{2} \beta_{0} .
$$

Letting $N=N_{s} e^{-c h^{\prime}}$,

$\int_{0}^{1} N d s=N_{s} \int_{0}^{R_{0}} \exp \left\{-c\left[s^{2} \frac{\cos ^{2} \beta_{0}}{2 r_{0}}+s \sin \beta_{0}\right]\right\} d s$.

Integrating (A6),

$\int_{0}^{1} N d s=\frac{N_{s}}{c} \frac{e g^{2} \sqrt{\pi} g}{\sin \beta_{0}}\left[\operatorname{erf}\left(R_{0} \cos \beta_{0} \sqrt{\frac{c}{2 r_{0}}}+g\right)-\operatorname{erf}(g)\right]$,

where erf $(x) \equiv \frac{2}{\sqrt{\pi}} \int_{0}^{x} e^{-t^{2}} d t$

$$
\text { and where } g=\sqrt{\frac{c r_{0}}{2}} \tan \beta_{0} \text {. }
$$

\section{References}

Bean, B. R., and G. D. Thayer, Models of the atmospheric radio refractive index, Proc. IRE 47, 740-755 (May 1959). Bean, B. R., and G. D. Thayer, CRPL exponential reference atmosphere, NBS Mono. 4 (Oct. 1959).

Hoehndorf, F. W., Accuracy limits due to refraction on electronic tracking of space vehicles. AFOSR Tech. Rept. 107 (Apr. 1961). 